\title{
DETERMINISTIC PERIODICAL AND QUASIPERIODICAL SURFACES OF III-V COMPOUNDS: PREPARATION, INVESTIGATIONS AND APPLICATIONS
}

\author{
N.L. DmitruK*, O.I. MaYeva, O.B. Yastrubchak and G.V. Beketov \\ Institute of Semiconductor Physics, National Academy of Sciences \\ 45 Prospect Nauki, 252028 Kiev-28, Ukraine
}

Autocorrelation functions for rough (random, quasiperiodical and deterministic-periodical) surfaces are deduced from the surface profiles determined by using the line-by-line analysis of atomic force microscopy images. It is shown that the initial parts of autocorrelation functions have a Gaussian form. An attempt to use the concept of fractal as a bridge between deterministic periodic and random (spontaneous) surfaces including quasiperiodic ones have been made.

PACS numbers: $68.35 . \mathrm{Bs}, 73.40 . \mathrm{Ns}, 78.30 . \mathrm{Fs}$

\section{Introduction}

The corrugated surfaces of semiconductors are often used to construct high sensitive photodetectors or solar cells. We have previously shown $[1,2]$ that the significant enhancement of the efficiency for GaAs or InP based photodetectors within the wide spectral range may be obtained using microrelief (quasiperiodical) anisotropically etched surfaces. A controlled excitation of the surface plasmon-polaritons (SPPs) in the visible has become possible via periodic surface structures (gratings). SPP waves excited in thin metal film-semiconductor structure provide selective coupling of light into semiconductor with increased quantum effciency and with other unusual functional properties. For the photodetectors with quasiperiodical (spontaneous corrugation) and especially with periodically corrugated (grating) interface SPP excitation is an additional source of photosensitivity enhancement and affects their light polarization sensitivity. With a suitable choice of grating parameters (groove depth and period) a strong coupling of polarized light can be achieved.

In connection with that the problem of interaction of optical waves with rough surfaces has been of a greatly intensified interest. Surface roughness can

*e-mail: nicola@dep39.semicond.kiev.ua 
strongly modify optical effects that exist in its absence. Several types of rough surfaces that give rise to the above-mentioned effects, have been considered. They can be deterministic and periodic, such as one-dimensional grating; they can be spontaneous both quasiperiodic (quasigrating), and random (dendrites). These types of rough surfaces can be defined through the statistical properties of the surface profile function. The statistical parameters, namely, root mean square (rms) value of the surface roughness $\delta$, and autocorrelation length (ACL) $\sigma$, determined from the autocorrelation function (ACF) can characterize the surface roughness. It is known that the introduction of fractal geometry [3] provides a new tool to describe naturally occurring rough surfaces. We have made an attempt to use the concept of fractals [4] as a bridge between deterministic periodic and random (spontaneous) surfaces including quasiperiodic ones. Besides an accurate and complete characterization of the surface roughness, the purpose of such study is to test the validity of such a treatment on deterministic surfaces. The results reported here were obtained on the rough surfaces of III-V semiconductors (GaAs, InP), some of which intended for the use in the sensor applications. With these applications in mind we intend to test the effect of the surface characteristics on the photosensitivity enhancement where both random and periodic surfaces are considered.

\section{Fabrication of rough surfaces and experimental procedure}

Various surface topographies can be obtained from different conditions of preparation. In particular, the adjunction of a suitable chemical agent, duration and temperature to the etching solution provides surfaces with different morphology and various scales of roughness. The surface microrelief of spontaneous dendrite type has been obtained by anisotropic chemical etching in concentrated $\mathrm{HNO}_{3}$ (GaAs) or $\mathrm{H}_{2} \mathrm{SO}_{4}(\mathrm{InP})$. To prepare the microrelief of grooves type (quasiperiodical) concentrated $\mathrm{HCl}$ for $\mathrm{InP}$ or $\mathrm{HF}: \mathrm{H}_{2} \mathrm{SO}_{4}: \mathrm{H}_{2} \mathrm{O}_{2}$ (2:2:1) mixture for GaAs was used. The fabrication of the diffraction grating was performed by holographic exposure of semiconductor substrates to dilute etchant solution with a blue laser $(488 \mathrm{~nm})$. The microstructure of each relief surface was revealed by both the SEM and the AFM techniques.

Thermally evaporated metal ( $\mathrm{Ag}, \mathrm{Au}, \mathrm{Al}$ ) films were deposited at room temperature through shadow mask to form surface barrier structures (SBSs) of Schottky type. These films were not annealed. For reflection measurements the samples were completely covered by metal. The experimental setup allowed measurements of spectral and angular characteristics of both the SBSs short-circuit photocurrent $i_{\mathrm{ph}}$ and light reflection of microrelief surface within the wavelengths range of $\lambda=400 \div 1000 \mathrm{~nm}$.

\section{Statistical and fractal model of rough surfaces}

Before the presentation of experimental results it may be useful to remind mathematical descriptions of rough surfaces we use. The two main statistical parameters which characterized random rough surface, namely, the ACL $\sigma$ and the rms $\delta$ are deduced from the line-by-line reconstructed relief of the surface considered through the $\mathrm{ACF}$. The method described provides the use of random, periodic, and fractal functions to model rough surfaces. 
A microrelief surface centred on the plane $z=0$ with height fluctuations $\delta z(x)$ as a function of the lateral co-ordinate $x$ may be described by the so-called autocorrelation function $G(X)$ as follows [5]:

$$
G(X)=\langle\delta z(x) \delta z(x+X)\rangle=\delta^{2} \exp \left[-(X / \sigma)^{2 h}\right] .
$$

Depending on the morphology of the roughness the value of the roughness exponent $h$ is:

(1) small, $h \Rightarrow 0$, if the surface is sharp and jagged;

(2) $h \Rightarrow 1$ for more gently rounded surfaces (Gaussian type);

(3) $h \approx 1 / 2$ corresponds to the case of random-walk fluctuations ("exponential" surface).

From Eq. (1) we have

$$
\ln \ln (G(0) / G(X))=2 h \ln (X / \sigma)
$$

i.e., the scaling exponent $h$ can be calculated from the slope of linear dependence (2).

Besides, we shall use the fractal function as a bridge between deterministic periodic and random functions [4]; then geometrical and scattering characteristics of surface are controlled by its fractal dimension. This fractal function, which we use to model a surface with one-dimensional roughness, is expressed as a weighted sum of periodic functions

$$
z(x)=\sigma C \sum_{n=0}^{N-1}(D-1)^{n} \sin \left(K_{0} b^{n} x+\varphi_{n}\right),
$$

where $1<D<2$ is the roughness fractal dimension, $K_{0}$ is the fundamental spatial wave number, $b(>1)$ is spatial frequency scaling parameter, and $N$ is the number of tones. The traditional parameters used in random surface modeling are rms $\delta$, correlation length $\sigma$, and rms slope $\sigma_{\mathrm{s}}$. The correlation length $\sigma$ of this model can be found with the aid of the autocorelation functions

$$
G(X)=\delta^{2} \frac{\left[1-(D-1)^{2}\right]}{\left[1-(D-1)^{2 N}\right]} \sum_{n=0}^{N-1}(D-1)^{2 n} \cos \left(K_{0} b^{n} X\right) .
$$

It is of interest to find the relation between two sets of parameters.

\section{Experimental results and discussion}

AFM-technique was used to obtain the surface geometric statistics. The method [6] allows us: (i) to reconstruct the surface profile line-by-line and thus one obtains a grid of $n$ (20) scanning lines with $m(250)$ points per line and (ii) to compute from these data the ACF that statistically describes the surface. From the various fits to the experimental ACF we carried out, we present two best of them, which are given in Table. With the greater number of adjustable parameters in fitting procedure we were able to improve the fit (column $A$, see Table). As for dendrite surface it is isotropic and it is then quite realistic to consider a one-dimensional model. The initial portion of the ACF for such random surface is fairly close to Gaussian. The values of $\delta$ deduced from ACF (through the line-by-line analysis) are in agreement with the values of $\delta$ estimated from 
AFM-image immediately. As an illustration, Fig. 1 shows both the ACF for rough surface and the corresponding AFM-surface image. As seen in Fig. 1, the relief formed in the concentrated $\mathrm{HCl}$ is two-dimensional and has characteristics of both random roughness and periodic roughness structure. In this case simple one-argument functions can be considered, which are used for relief description corresponding to the measurements across the grooves. The ACF then has also a Gaussian form. Beyond the Gaussian part ACF oscillates with a pseudoperiodicity. This type of surface (intermediate between periodic and perfectly random surfaces) should play a specific role in light scattering and related problems.

TABLE

Characteristics of the rough surfaces.

\begin{tabular}{l|c|c|c|c|c|c|c}
\hline \hline Roughness & \multirow{2}{*}{$\begin{array}{c}\text { G(0) } \\
\text { type }\end{array}$} & \multicolumn{2}{|c|}{$(\mathrm{A}) \ln (\ln [G(0) / G(X)])$} & \multicolumn{3}{|c}{$(\mathrm{B})$ Fractal } \\
\cline { 3 - 8 } & {$[\mathrm{nm}]$} & $\sigma[\mathrm{nm}]$ & $h$ & $\delta[\mathrm{nm}]$ & $K_{0}\left[\mathrm{~nm}^{-1}\right]$ & $D$ & $b$ \\
\hline dendrite & 835 & 661 & 0.927 & 829 & 0.00093 & 1.81 & 1.43 \\
quasigrating & 726 & 208 & 0.959 & 728 & 0.00382 & 1.67 & 1.58 \\
grating 1 & 57.7 & 914 & 0.915 & 57.2 & 0.00149 & 1.21 & 2.03 \\
grating 2 & 28.8 & 992 & 0.869 & 28.8 & $\mathbf{0 . 0 0 1 3 2}$ & 1.40 & 1.71 \\
grating 3 & 61.8 & 125 & 0.985 & 61.4 & 0.00774 & 1.33 & 1.96 \\
grating 3+Al & 55.7 & 105 & 1.048 & 56.1 & 0.00616 & 1.48 & 1.74
\end{tabular}
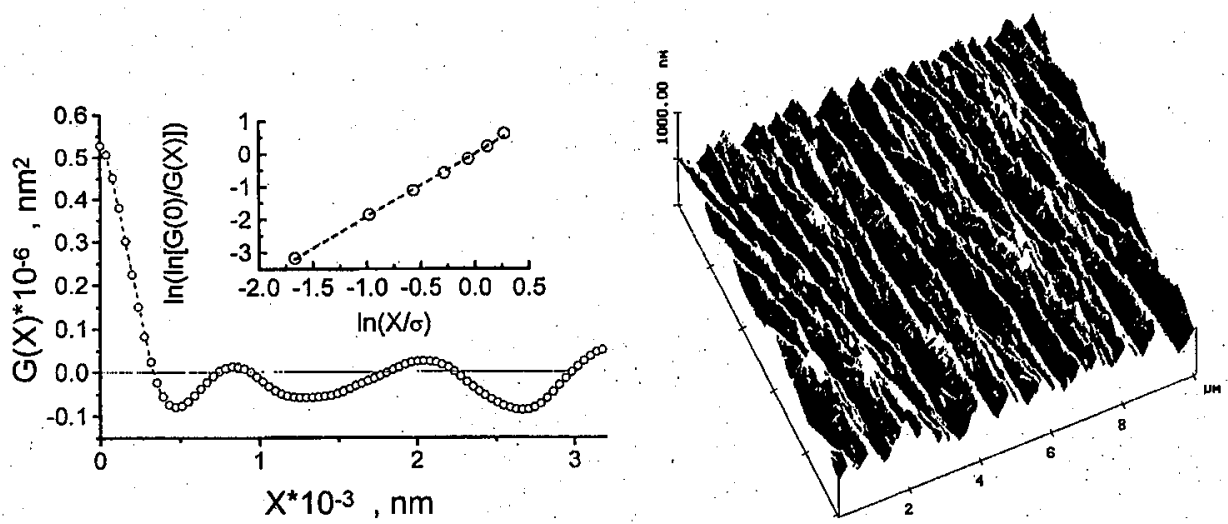

Fig. 1. ACF related to the quasiperiodical surface relief of GaAs. The inset shows the dependence of $\ln (\ln [G(0) / G(X)])$ on $\ln (X / \sigma)$. The AFM-image of quasigrating is shown on the right of the figure.

The data of Table show that a fractal model including only a finite range of spatial frequencies $(N=6)$ allows us to describe real microrelief surfaces. Indeed, the fractal dimension $D$ is a measure of the roughness: the deterministic character of relief increases as the fractal dimension $D$ decreases. The value of the spatial-frequency scaling parameter $b$ also increases in the direction of the increase 
in the degree of surface order. The values of $\delta$ and $K_{0}=2 \pi / \Lambda_{0}\left(\Lambda_{0}\right.$ is the main period of relief) are consistent with AFM-images.

\section{Application of rough surfaces}

This section describes a possibility of application of rough surfaces to construct photodetectors and sensors, the principle of which is based on using or detection of a surface plasmon resonance (SPR). The SBSs (photodetectors) with quasiperiodical microrelief at the interface (Fig. 2a, curves $3,3^{\prime}$ and $4,4^{\prime}$ ) show a supplementary photocurrent $\left(i_{\mathrm{ph}}\right)$ rise within the $\Theta$-region, whose polarization angular characteristics are evidence for its polariton nature, in contrast to the cases of structures with flat (Fig. 2a, curves 1, $1^{\prime}$ ) or random microrelief surface (Fig. 2a, curve $2,2^{\prime}$ ). The changing of the maximum in $p$-polarization $i_{\mathrm{ph}}^{p}$-curve location is consistent with wavelength variation (see curves 3 and 4 in Fig. 2a). Indeed, the
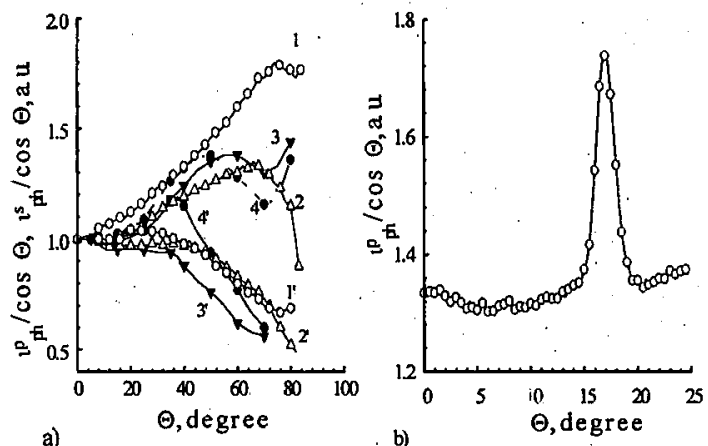

Fig. 2. (a) The angular dependences of photocurrent $i_{\mathrm{ph}}^{p, s} / \cos \Theta$ of Ag-InP photodetectors with flat $\left(1,1^{\prime}\right)$, random $\left(2,2^{\prime}\right)$ and quasiperiodical $\left(3,3^{\prime} ; 4,4^{\prime}\right)$ microrelief surface for the light of $p-(1-4)$ and $s-\left(1^{\prime}-4^{\prime}\right)$ polarization; $\lambda$, nm: $550\left(1-3,1^{\prime}-3^{\prime}\right), 750\left(4,4^{\prime}\right)$. (b) Initial part of angular dependence of $i_{\mathrm{pl}}^{p} / \cos \Theta$ of Ag-InP photodetector with deterministic (grating) microrelief surface for the $p$-polarized light; $\lambda=750 \mathrm{~nm}$.

resonant excitation of surface plasma polaritons on the air-metal $(\mathrm{Ag})$ interface with periodical microrelief (grating) (Fig. 2b) leads to a rise in SBSs photocurrent under phase synchronism conditions [7]: $K_{\mathrm{SPP}}=m(2 \pi / a)+(\omega / c) \sin \Theta$, where $K_{\mathrm{SPP}}$ is the wave vector of the SPP and $m= \pm 1,2 \ldots$, an integer and $a$ is the grating period. If the diffraction grating vector is oriented such that it is out of the plane of incidence then SPR can be used to provide a strong degree of polarization conversion of radiation. By scanning the angle of incidence for fixed wavelength using crossed polarizers between the radiation source and the photodetector, a strong resonance maximum at the SPP excitation angle is achieved (Fig. 3). The angle of this resonance is extremely sensitive to variations in the dielectric constant of the medium in contact with the surface of the metal film. Changes in this peak angle value are a measure of changes in the SPR due to the environment. Thus, a grating with evaporated metal (Al) film may be used as a main part of sensor (Fig. 3). 


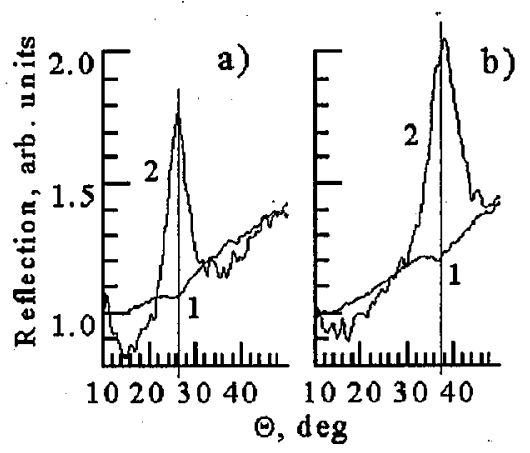

Fig. 3. Normalized to signal at $\Theta=7^{\circ}$ experimental curves for SPR on Al-GaAs structure with diffraction grating (period $650 \mathrm{~nm}$, modulation height $11 \mathrm{~nm}$ ): the measurement of both $p$-polarized (1) and $s$-polarized (2) reflections with $p$-illumination: $\lambda=850 \mathrm{~nm}(\mathrm{a}) ; \lambda=900 \mathrm{~nm}$ (b). The azimuthal angle of the grating is $45^{\circ}$.

\section{Conclusions}

We elaborate technology of wet chemical etching preparation of relief surfaces of III-V semiconductors allowing to obtain both random and deterministic surfaces of diffraction grating type. The concept of fractal functions is used for the description of peculiarities of rough surface morphology. Autocorrelation function of three types of real rough surfaces was determined by fitting experimental data in the frame of the fractal model. The application of relief surfaces of GaAs and InP as a base of both polariton optoelectronic photodetectors and convertors of polarized electromagnetic radiation is described, too.

\section{References}

[1] E.V. Basiuk, N.L. Dmitruk, O.I. Mayeva, Semiconductors 27, 232 (1993).

[2] N.L. Dmitruk, O.Yu. Borkovskaya, O.I. Mayeva, O.V. Fursenko, Microelectron. J. 27, 37 (1996).

[3] B.B. Mandelbrot, The Fractal Geometry of Nature, Freeman, New York 1982.

[4] D.L. Jaggard, X. Sun, J. Opt. Soc. Am. A 7, 1131 (1990).

[5] S.K. Sinha, Acta Phys. Pol. A 89, 219 (1996).

[6] F. Varnier, N. Mayani, G. Rasigni, J. Vac. Sci. Technol. A 7, 1289 (1989).

[7] A.A. Maradudin, in: Modern Problems in Condensed Matter Sciences, Surface Polaritons, Eds. V.M. Agranovich, A.A. Maradudin, North-Holland, Amsterdam 1982, p. 405. 